\title{
Imaging Meso-Scale Structures in TEXTOR with 2D-ECE
}

\author{
I.G.J. CLASSEN, R.J.E. JASPERS, H.K. PARK ${ }^{1)}$, G.W. SPAKMAN, M.J. VAN der POL, \\ C.W. DOMIER ${ }^{2)}$, A.J.H. DONNE, N.C. LUHMANN Jr${ }^{2)}$, E. WESTERHOF, M.W. JAKUBOWSKI ${ }^{3)}$ \\ and the TEXTOR team ${ }^{3)}$ \\ FOM Institute for Plasma Physics Rijnhuizen, Association Euratom-FOM, Partner in the Trilateral Euregio Cluster \\ Nieuwegein, Netherlands \\ 1) Princeton Plasma Physics Laboratory, Princeton, New Jersey, U.S.A \\ ${ }^{2)}$ University of California at Davis, Dept. of Applied Science, Davis, California, U.S.A \\ ${ }^{3)}$ Forschungszentrum Jülich, Institut für Plasmaphysik, Association EURATOM-FZJ, Partner in the Trilateral Euregio \\ Cluster, D-52425 Jülich,Germany
}

(Received 1 December 2006 / Accepted 3 March 2007)

\begin{abstract}
The detection and control of instabilities in a tokamak is one of the exciting challenges in fusion research on the way to a reactor. Thanks to a combination of an innovative 2D temperature imaging technique (ECEI), a versatile ECRH/ECCD system and a unique possibility to externally induce tearing modes in the plasma, TEXTOR is able to make pioneering contributions in this field. This paper focuses on two meso-scale phenomena in tokamaks: $m=2$ tearing modes and magnetic structures in the stochastic boundary. In these cases the 2DECEI diagnostic can resolve features not attainable before. In addition the possibility to use the diagnostic for fluctuation measurements is addressed.
\end{abstract}

(C) 2007 The Japan Society of Plasma Science and Nuclear Fusion Research

Keywords: Electron cyclotron emission, plasma diagnostic, imaging, magnetic island, fluctuations, suppression, stochastic fields, electron cyclotron heating, tearing mode

DOI: $10.1585 / \mathrm{pfr} .2 . S 1031$

\section{Introduction}

The control of MHD structures is one of the challenges in fusion research: the suppression of neoclassical tearing modes by electron cyclotron current drive (ECCD) to operate the reactor close to its stability limit or the mitigation of ELMs by ergodisation of the plasma edge [1] are just two major examples showing the importance of this field.

On TEXTOR a coherent programme is executed around this theme, based on three pillars: an innovative 2D electron cyclotron emission imaging (ECEI) diagnostic to image the MHD structures, a versatile ECRH/ECCD system to manipulate these and finally a set of perturbation coils to induce such structures.

The potential for an ECEI diagnostic has been demonstrated by investigating the sawtooth crash [2]. This paper will concentrate on three different aspects: i) control of the $m=2$ tearing mode; ii) visualization of the magnetic structures induced in the plasma edge by the perturbation coils; and iii) temperature fluctuation patterns. Before focusing on the details of these studies a short overview of the ECEI system is at its place, together with some remarks on TEXTOR and the used hardware.

\section{The Experimental Tools}

Although TEXTOR is a limiter tokamak with a circular cross-section (major/minor radius $R_{0}, a=1.75,0.46 \mathrm{~m}$, and typically $I_{\mathrm{p}}=350 \mathrm{kA}, B_{\mathrm{t}}=2.25 \mathrm{~T}$ ), some of its tools are completely unique, allowing for an exciting research programme. The relevant ones here are the ECEI system and the dynamic ergodic divertor, DED.

\subsection{Electron cyclotron emission Imaging- ECEI}

The 2D-ECEI [3] consists of a 16 element vertical array of heterodyne receivers, which, combined with wide aperture optics, can image a vertical slice of approximately $16 \mathrm{~cm}$ in the plasma. Since each receiver element is treated as a conventional second harmonic $\mathrm{X}$ mode ECE radiometer with 8 frequency channels, corresponding to a radial coverage of about $7 \mathrm{~cm}$. The system in total can image an area of $16 \times 7 \mathrm{~cm}^{2}$ on an array of $16 \times 8$ sampling volumes, centered around the mid-plane, see Fig. 1. The radial location can be shifted by either changing the local oscillator frequency (tunable from 85 to $130 \mathrm{GHz}$ ) or by varying the magnetic field. The resolution amounts to $2 \mathrm{~cm}$ in the vertical direction as limited by the optics and $1 \mathrm{~cm}$ radially. The time resolution is up to $500 \mathrm{kHz}$.

\subsection{Dynamic Ergodic Divertor}

The Dynamic Ergodic Divertor (DED) on TEXTOR consist of a set of 16 helical perturbation coils on the high 


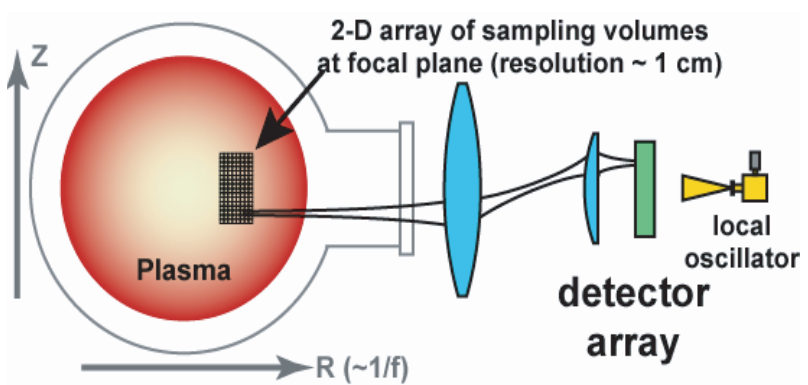

Fig. 1 Sketch of the ECE-I system, consisting of an $16 \times 8$ array of sampling volumes in the plasma.

field side, with a helicity aligned with the $q=3$ field lines [4]. The DED can be operated DC and AC up to $10 \mathrm{kHz}$. It can be used to generate a perturbation field with a dominant $12 / 4,6 / 2$, or $3 / 1$ mode structure. When operated in its $3 / 1$ mode it possesses a strong $2 / 1$ side band, which, above a certain threshold, triggers an $m=2, n=1$ tearing mode. In the experiments reported here $1 \mathrm{kHz}$ AC currents are used, resulting in a $1 \mathrm{kHz}$ toroidal rotation for the $m / n=2 / 1$ island.

Operation of the DED in the $12 / 4$ mode will mainly disturb the plasma edge. In that case, the plasma edge consists of an ergodic region, arising from the overlap of neighbouring island chains at resonant surfaces $(q=10 / 4$, $11 / 4, \cdots)$. Close to the edge, open field lines with a very short wall-to-wall connection length arise, the laminar zone. The result is a complicated magnetic structure. In particular, long thin ergodic 'fingers' surrounded by laminar regions, connect the ergodic region with the wall.

\section{Suppression of $m / n=2 / 1$ Tearing Modes}

Tearing modes can be induced externally at TEXTOR by the DED, allowing detailed investigations in the tearing mode physics. Here, we concentrate on the suppression of these modes. This can be done in well defined experimental conditions: the perturbation amplitude can be externally governed, the rotation of the perturbation field and the rotation of the plasma can be independently controlled and finally the gyrotron is able to deposit locally its power inside the island or drive current in the island. The excitation of the mode depends on the strength of the perturbation current, the relative rotation between the perturbing field and the plasma, on density and beta [5].

Once the mode is excited the ECRH/ECCD system $(140 \mathrm{GHz}, 800 \mathrm{~kW}, \mathrm{CW}$ or modulated, poloidal and toroidal movable launcher [6]) can be applied to test the scenarios for island suppression. Various systematic scans of the mode suppression ratio have been performed, in order to compare (i) ECRH with ECCD, (ii) CW heating with pulsed heating at various duty cycles and (iii) the difference between heating in the $\mathrm{X}$ - and $\mathrm{O}$-points of the island.

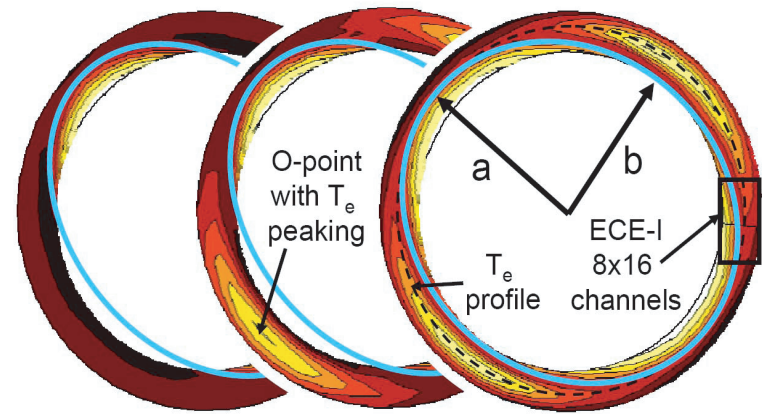

Fig. 2 Poloidal reconstructions of the island, showing the time evolution during the suppression process: a) flat island b) heated island c) suppressed island. The color coding goes from dark (cold) to light (hot).

Strongest suppression is found when pulsed ECRH is applied within the O-point of the island [7].

To interpret the physics mechanism at work in suppressing the island, quantitative information on the evolution of the temperature is required. To visualize the ECEI data in an easily interpretable way, a poloidal reconstruction is used in which the data for one full rotation period are mapped onto a poloidal shell, assuming rigid plasma rotation. Note that this reconstruction only represents the low field side structure of the island. Figure 2 shows the reconstruction of the island during the three main stages of the suppression process [8]. The first stage is the situation in which the island has been generated and has reached a saturated width of about $12 \mathrm{~cm}$, before ECRH was switched on. In this phase, the $T_{\mathrm{e}}$ profile inside the island is still flat. The hot, central plasma, approximately elliptically deformed by the island, is clearly visible. The second reconstruction, shortly (about $10 \mathrm{~ms}$ ) after switch on of ECRH, shows a peaked $T_{\mathrm{e}}$ profile inside the island. The third stage is the steady state situation long (more than $100 \mathrm{~ms}$ ) after switch on of ECRH. The island is now suppressed to about half the initial size and the central plasma is less deformed and the peaked temperature region inside the island is narrower.

An automatic algorithm is used to extract the main island parameters, its width and amplitude, from the ECEI data. The time evolution of these parameters is given in Figure 3. Knowing the $T_{\mathrm{e}}$ profile and the power deposited inside the island, the diffusivity $\chi_{\mathrm{e}}$ in the island can directly be calculated from simple power balance calculations. It turns out that $\chi_{\mathrm{e}}$ is $1-1.5 \mathrm{~m}^{2} / \mathrm{s}$, close to the value found for the ambient plasma, which amounts to $1 \mathrm{~m}^{2} / \mathrm{s}$ [8].

The evolution of the island width is governed by the modified Rutherford equation. For the special case under consideration here, the island evolution is in addition to the tearing stability index $\Delta^{\prime}$ expanded by a destabilizing term due to the action of the DED (giving rise to shielding currents inside the island to counteract) and a stabilizing term due to the heating effect of the ECRH (the reduced resistiv- 


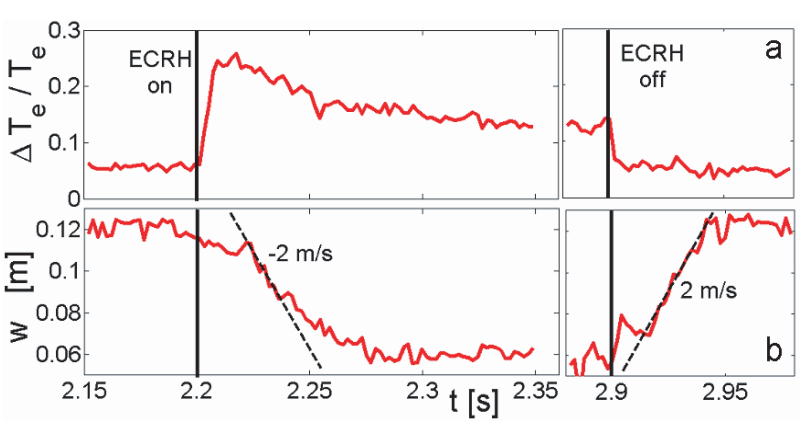

Fig. 3 Typical evolutions of the temperature peaking inside the island (a) and the island width w (b) after switching on and off of ECRH, in case of island excitation by the DED. Note that the dashed line is the calculated result for the ECRH term according to eq. 1, in good agreement with the data.

ity allows for an enhancement of the induced current inside the island). The mathematical expression for this modified Rutherford equation then looks like:

$$
0.82 \tau_{r} \frac{d w}{d t}=r_{s}^{2} \Delta^{\prime}+M_{D E D}-M_{E C R H}
$$

where $\tau_{\mathrm{r}}$ is the current diffusion time, $w$ the island width, and $r_{s}$ the resonant surface. The DED term $\left(M_{D E D}=\right.$ $\left.2 m r_{s}\left(w_{v a c} / w\right)^{2}\right) ; w_{v a c}$ being the vacuum island width, about $4 \mathrm{~cm}$ for this case) is fully known and the ECRH term can be evaluated numerically using the measured $T_{\mathrm{e}}$ data inside the island. Finally the $\Delta^{\prime}$ term can be estimated from the balance in the saturated state. Thus this TEXTOR experiment is unique in the sense that it can separate single terms in the Rutherford equation. The dashed line in Fig. 3 represents the theoretical expectation from the right hand side of Eq.1 (the $M_{\mathrm{ECRH}}$ term), and is in good agreement with the experimental data.

The stabilizing effect of heating on the island evolution can thus be calculated, and it appears to be the dominating effect in these experiments. Heating may well play a role in Neoclassical Tearing Mode (NTM) stabilization in ITER as well and, consequently, result in lower power requirements for this in ITER [8].

\section{Structures in the Ergodic Edge}

Another area where ECEI provided valuable information is in imaging edge structures induced by the DED in the 12/4 mode. In Fig. 4a-d ECEI measurements at the low field side (LFS) plasma edge during a shot with DC DED are presented. During this phase of the shot the plasma current was ramped down from $I_{\mathrm{p}}=390-365 \mathrm{kA}$. The data is normalized to the temperature profile just before switching on the DED, and hence reflects the relative change during DED operation. With DED, the temperature drops over the entire observation volume of ECEI, which is a clear sign of an enhanced transport level. The measurements also show structures that evolve with the changing plasma current.
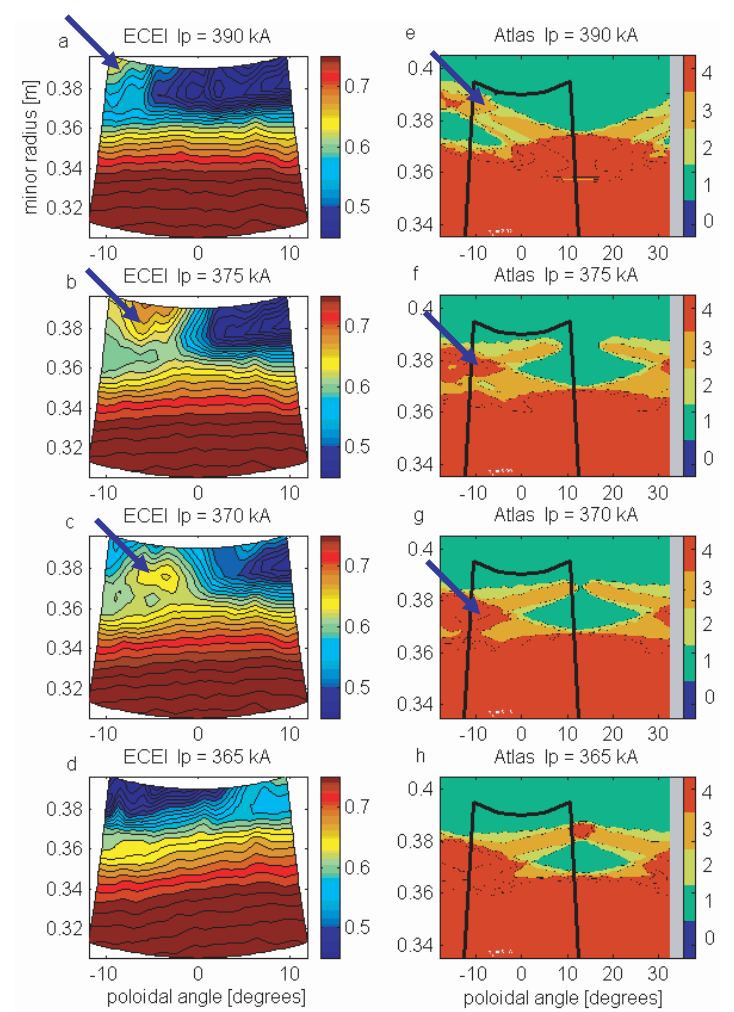

Fig. 4 Comparison of ECEI temperature measurements with Atlas calculations [9] of the connection length (laminar plots) during a plasma current ramp. The hotter region is indicated by the arrows.

Due to the changing q profile the DED induced structures are expected to evolve. Figures $4 \mathrm{e}-\mathrm{h}$ give the corresponding 'laminar plots', as calculated with the Atlas code [9]. A laminar plot is a contour plot of the wall-to-wall connection length in units of poloidal turns. The laminar zone consists mostly of the 1 and 2 poloidal turn field lines. The field lines with connection length longer than 4 poloidal turns belong to the ergodic region. The black contour indicates the observation area of ECEI. Fig. 4e-h show the inward migration of a structure of ergodic plasma, as corroborated by the (relative) 'hot' temperature region observed in the ECEI data (denoted by arrows in Fig. 4).

In Fig. 5a ECEI data from a shot with DC DED is shown. The data clearly shows a relatively hot region separated from the main plasma by a colder region, similar to the structure in Fig. 4. In Fig. 5b, estimates of the absolute temperature profiles at cross sections 1 and 2 indicated in Fig $5 \mathrm{a}$ are shown, along with the poloidally averaged profile and the non-DED reference. The profiles clearly show the overall drop in temperature and the large poloidal variation in profile shape during DED. The extent of the DED affected region is clearest seen in Fig. 5c, where the ratio between the temperature gradient in the non-DED reference case and the temperature gradient during DED is plotted. Assuming heat flux and density profiles stay the same this ratio is a measure for the enhancement of the 

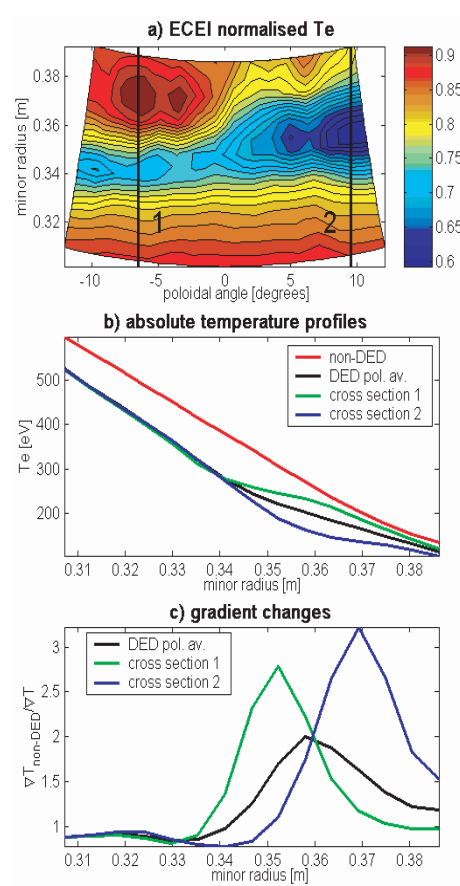

Fig. 5 Comparison of $T_{\mathrm{e}}$ profiles during DED with non-DED reference.

heat transport during DED [10]:

$$
\frac{\nabla T_{e, n o n-D E D}}{\nabla T_{e, D E D}} \approx \frac{\chi_{e r g}}{\chi_{\perp}}
$$

Where $\chi_{\perp}$ is the perpendicular diffusion coefficient in the unperturbed case and $\chi_{\text {erg }}$ is the effective heat diffusion coefficient in the perturbed magnetic field. Fig. $5 \mathrm{c}$ indicates a significant transport enhancement in the plasma regions where the laminar zone is expected. In the ergodic region (for example in the 'hot spot') transport levels are close to the unperturbed values.

\section{Fluctuations}

The final example of the capabilities of the ECEI system is fluctuations. Previously reported measurements with the TEXTOR O-mode reflectometer [11] have shown density fluctuations near the plasma edge, the quasi-coherent mode. This mode provides a test bed for the ECEI system.

The cross correlation function of two ECE channels indicates a clear $90 \mathrm{kHz}$ oscillation around zero time lag. A bandwidth of around $50 \mathrm{kHz}$ can be derived from the correlation function, in agreement with reflectometer measurements at the same radius. The contour plot in Fig. 6 shows the cross correlation function as a function of poloidal channel separation $\Delta \mathrm{s}$. From the phase shift as a function of $\Delta \mathrm{s}$, a poloidal velocity of about $6 \mathrm{~km} / \mathrm{s}$ can be deduced. The poloidal wavelength can be estimated to be around $7 \mathrm{~cm}\left(\lambda_{\mathrm{pol}}=v_{\mathrm{pol}} / f\right)$, from which a poloidal mode number around $m=40$ is found.

The fluctuation amplitude however is affected by the optical thickness $\tau$, which is low ( $\tau$ is about 1.5) at the

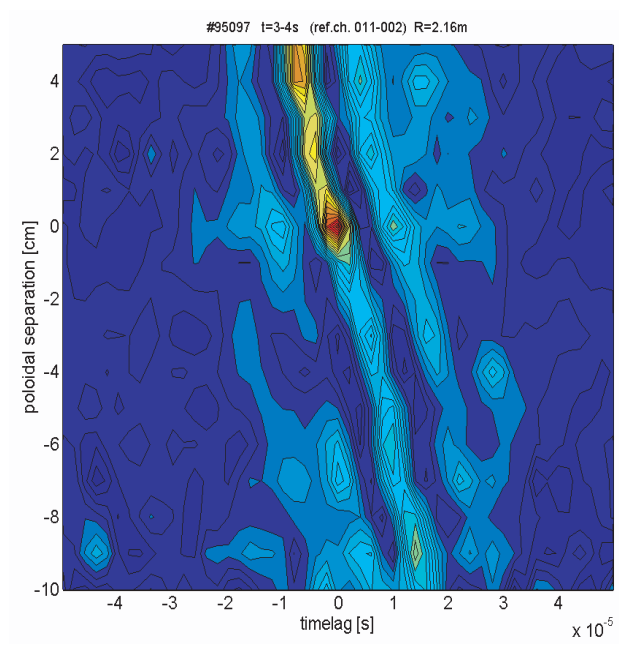

Fig. 6 Cross correlation spectrum for the poloidal evolution of ECEI fluctuation measurements of the quasi coherent mode.

outboard side of the observation volume, rapidly increasing towards the plasma centre. Hence, in fact a mixture of density and temperature fluctuations is measured. The density fluctuation amplitude however is known from reflectometer data [11], so the density contribution to the measured radiation temperature fluctuations can be estimated and corrected for, just as for the optical thickness. This has been done and the temperature fluctuation amplitude amounts to be about $1 \%$ near the edge, decaying more inward, falling below the detection limit of $0.1 \%$ after $6 \mathrm{~cm}$.

From these investigations one can conclude that a) ECEI is able to measure the fluctuations down to a level of $0.1 \%$ and $b$ ) the quasi-coherent mode exhibits apart from density also temperature fluctuations.

\section{Conclusion}

In this paper the powerful capability of the high resolution $2 \mathrm{D} T_{\mathrm{e}}$ imaging data from the ECEI diagnostic has been demonstrated in three examples, where the ECEI data were crucial for the interpretation and understanding of the physics mechanisms at play: i) the dominant effect of heating inside the island in the suppression of the $m=2$ tearing mode; ii) the verification of structures induced by the DED in the plasma edge and iii) temperature fluctuation in quasi coherent modes.

Very recently the ECEI system at TEXTOR is being applied for the study of ELM's. Here the situation is even more complicated due to the interplay of different effects like optical thickness, cut-off effects, refraction etc. However, potentially ECEI could provide new insights in this phenomenon. Attempts at TEXTOR are therefore being undertaken to study the interaction of the DED induced perturbation field with the ELM appearance, a promising technique presently being pursued on different devices for ELM mitigation [1]. 
An upgrade of the ECEI system is foreseen which will add a second array to the system. This will allow measuring either an extended range of the plasma, or to compare high field side with low field side emission, correlate core with edge or focus on mode coupling events such as the triggering of the neoclassical tearing mode by sawtooth crashes. In conclusion: A wealth of exciting physics is still to be discovered with this powerful system.

\section{Acknowledgments}

This work, supported by the European Communities under the contract of Association between EURATOM/FOM, was carried out within the framework of the European Fusion Program with the ECEI system developed under US DOE funding. The views and opinions expressed herein do not necessarily reflect those of the European Commission.

[1] T.E. Evans et al., Nature Physics 2, 419 (2006).

[2] H.K. Park et al., Phys. Rev. Lett. 96, 195003 (2006).

[3] H.K. Park et al., Rev. Sci. Instrum. 74, 4239 (2004).

[4] K.H. Finken K H (Ed.)., Special Issue: Dynamic Ergodic Divertor Fusion Eng. Design 37, 335 (1997).

[5] H.R. Koslowski et al., Plasma Phys. Contr. Fusion 48, B53 (2006).

[6] E. Westerhof et al., Nucl. Fusion 43, 1371 (2003).

[7] E. Westerhof et al., Nucl. Fusion, accepted for publ. (2007).

[8] I.G.J. Classen et al., Phys. Rev. Lett. 98, 035001 (2007).

[9] M.W. Jakubowski et al., Nucl. Fusion 44, S1 (2004).

[10] M. Kobayashi et al., Nucl. Fusion 44, S64 (2004).

[11] A. Krämer-Flecken et al., Nucl. Fusion 44, 1143 (2004). 